\title{
New Hitch Haiku: An Interactive Renku Poem Composition Supporting Tool Applied for Sightseeing Navigation System
}

\author{
Xiaofeng $\mathrm{Wu}^{1}$, Naoko Tosa ${ }^{1}$, and Ryohei Nakatsu ${ }^{2}$ \\ ${ }^{1}$ Academic Center for Computing and Media Studies, Kyoto University \\ Yoshida-Nihonmatsu, Sakyo, Kyoto, 606-8501 Japan \\ XiaofengWu@hotmail.com, tosa@media.kyoto-u.ac.jp \\ ${ }^{2}$ Interactive \& Digital Media Institute, National University of Singapore \\ Blk E3A \#02-04, 7 Engineering Drive 1, Singapore 117574 \\ idmdir@nus.edu.sg
}

\begin{abstract}
As is well-known, cultures are rooted in their unique regions, histories and languages. Communication media have been developed to circulate these cultural characteristics. As a part of our research "Cultural Computing", which means the translation of cultures using scientific methods representing essential aspects of Japanese culture[1], an interactive Renku poem generation supporting system was developed to study the reproduction of a traditional Japanese Renku by computer. This system extended the functionality of our previous Hitch-Haiku system to the Renku based on same association method and attached more cultural characteristics on it: the Renku verse displayed on the Japanese-style color pattern which represents the same season in Renku Kigo (seasonal reference) and the generated Renku verse including the information of sightseeing place.
\end{abstract}

Keywords: Haiku, Renku, Renku generation, Interactive art, Association.

\section{Introduction}

Haiku is a Japanese traditional poem style with minimal length of seventeen syllables (in Japanese) in three metrical phrases including a seasonal word called "Kigo." The original form of Haiku was called Hokku and in the late 19th century Shiki Masaoka revised it and finally established the present form of Haiku [3]. Haiku include various imaginative expressions and thus has been applauded by many people. Haiku is a story that generates context - the shortest story in the world. Known as the first great Haiku poet in the Japanese history, Matsuo Basho is responsible for "Oku No Hosomichi", a prime example of his work [4].

In 1959, Theo Lutz developed a system of a poem generation for the first time [5]. The system only showed words at random on grammatical rule, and could not generate a poem with user's interactions. In 1971, for the first time Masterman developed the generation system of a Haiku[6]. By rearranging the words which users chose from the pull down menu in the interaction process the system generates a Haiku. However, in these interactions, users could input only a few limited words into the system. 
In the field of Interactive Art or Game, the quality of contents is important, like "Passage Sets / One Pulls Pivots at the Tip of the Tongue" by Bill Seaman [7], and "An Anecdoted Archive from cold War" by George Legrady[8]. But from technologies viewpoint, only simple techniques have been used. On the other hands, in the field of AI, many researchers and developers have been using various kinds of techniques to find some relations among input words/phrases by users and to compose answers in relation to these inputs [9]. These techniques have been often used, because using one of these techniques they can develop an interactive system that can achieve relatively interesting interactions. But the relations they try to find out and they try to use in their systems are static, and the quality of their interactions have been mostly dependent on the quality of the relations given beforehand.

Based on his long carrier and an editor and a philosopher, Matsuoka defined four several basic forms called "Thoughtforms," as basic form of relations among things [10]. As the "Thoughtforms" can work as the basic method to re-construct interesting relations among words and enable to generate better Haiku, Tosa applied the technique to develop an interactive system, "Hitch Haiku", which supports a user for composing a Haiku [11]. The user only need to input some words into the system, and the system can compose phrases consisting of five-seven-five syllables which most fit to the user inputs. The system is called Hitch Haiku as it generates a Haiku "hitching" the phrases chosen based on the user inputs.

In order to extend this Hitch Haiku system and apply it to wider field, we adopt the success of the previous system, that is, generation of better quality Haiku using association method, and use more cultural features to develop a new interactive Renku composition supporting system which is applied for the Kyoto sightseeing navigation system.

\section{Concept}

The new Hitch Haiku system is designed to be a Renku composition supporting subsystem applied in the sightseeing navigation system which is based on our previous Hitch Haiku system, an interactive Haiku generation supporting system. Compared with the old Hitch Haiku system, there are three unique features in our new Hitch Haiku system:

(1) To generate Renku verse using searching and association method, the latter is the major point to get the better quality of Renku verse

The rules of Renku is much more restricted and complicated than those of Haiku, especially the limitation usage of Kireji in Renku creation. Based on the experience from previous experiment that the quality of the Haiku generated depends on the sensitivity of the user input words, we give up the steps of attaching Kireji to user input words to generate the phrases and try to generate the phrases of Renku verse in the way like human done, i.e., create the haiku phrase by the meaning of previous generated phrase and new keyword which can correspond to the previous generated phrase.

(2) During the generation of Renku verse, optionally we applied the Haiku thesaurus words related to the sightseeing place. Thus, it is possible to include the features of the specific sightseeing place into the Renku verse. 
(3) To show generated Renku verse on the Japanese-style color pattern, and furthermore, the selection of background color pattern is based on the representative season which corresponds to the Kigo of the generated Renku verse. Therefore, the user can feel the cultural characteristics not only from the phrases of Renku verse but also from the background color pattern which is also harmonious to the Renku verse.

By adding above features into our new Hitch Haiku system, we hope that the generated Renku verse and display style can show users more cultural characteristics.

\section{Process of Generation}

The Renku generation procedure in our new system is the process shown in follows (Fig.1). In details, the system works in the following steps:

(1) A user input his or her two favorite words (or phrases) in a text box of the Hokku (the first Haiku of the Renku) input webpage and select the sightseeing place in the Kyoto city from a optional selection box which including the most famous 110 hot spots of this city using mobile phone.

(2) The system searches the Haiku phrase databases both with and without Kigo and found out the phrases including one of the user input word and randomly select one. For Hokku, the system will do a special selection procedure: prior to select the phrase with Kireji. For the other Renku verses, because the Kirejis are forbidden, the system will select the phrase without any Kireji.

(3) In the other hand, the system searches the Haiku thesaurus word related to the user chosen sightseeing place. This Haiku thesaurus word will be used in the later Renku phrase generation.

(4) Once the first phrase was selected, a syntactic analysis for this phrase is carried out in the web server and the basic forms of noun or verb from each phrase are extracted.

(5) Then the system apply the elemental words of first phrase and the rest one of user input words to generate the second phrase of Renku verse by using association method. More in details, the system searches the words associated with the above elemental words and user input word, and extract the phrases including the association words from six types of databases in the system, which are Haiku thesaurus, Kigo thesaurus, idiom thesaurus, case frame of onomatopoeia, thesaurus, and case frame.

Furthermore, the system scores all phrases by weight: Haiku thesaurus is 3 , Kigo thesaurus is 3 , idiom thesaurus is 3 , case frame of onomatopoeia is 3 , thesaurus is 3 , case frame is 1 , user's relation is 5 . If a phrase includes two or more association words, the system sums the weight respectively. The system chooses one of the phrases with the highest weight.

(6) After generation the second phrase of Renku verse, the similar steps to (4) and (5) are executed again. The difference is this time, the system use the elemental words from the second phrase of Renku verse and the Haiku thesaurus word to do the association.

(7) Finally, the system "hitches" these three phrases to create the Renku verse. Obviously, the difference between old Hitch Haiku system and the new one is that the new system "hitches" phrases internally and natively. 


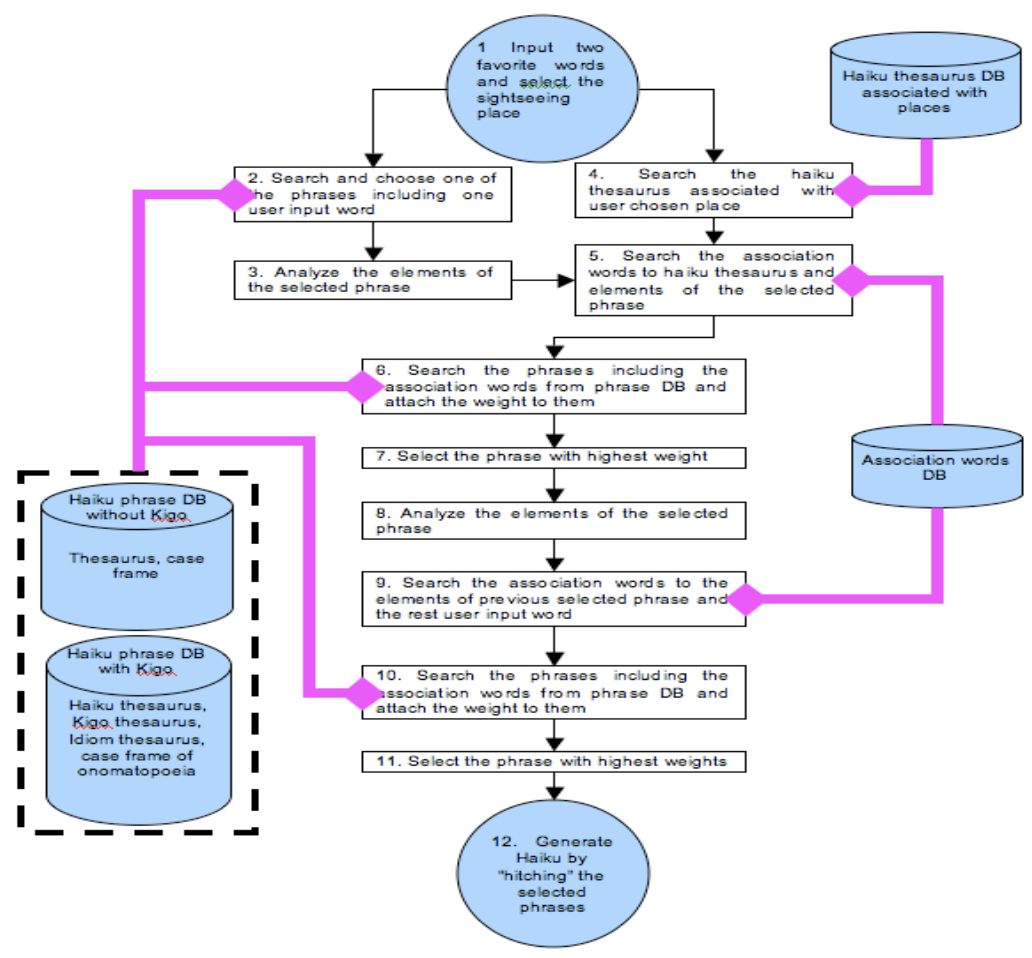

Fig. 1. Flowchart of Haiku generation algorithm

\section{Database}

In our new Hitch Haiku system, six types of databases, which are case frame database (about 31,000 records), thesaurus database (about 32,000 records), Haiku thesaurus database (about 2,500 records), Kigo thesaurus database (about 13,000), idiom thesaurus database (about 1,300 records) and the database of Case frame of onomatopoeia (about 8,800 records) are remained. But considering the requirements to generate Renku verse, for examples, the verse to describe the moon or flower in the specific season, we added more such information into these databases.

Furthermore, we added new Haiku thesaurus database for the sightseeing places in Kyoto city to show more local cultural background and local Haiku information which can integrate new Hitch Haiku system tightly to the sightseeing navigation system.

\section{Interaction Example}

With illustration figures listed below, we explain the abstract of usage of our system:

(1) A user log in the Hitch Haiku system with ID number (Fig.2).

(2) The user can select one of the functionalities provided by Hitch Haiku system by press the corresponding button (Fig. 3). 
(3) The user inputs the two favorite words into text boxes and chooses the hot spot from 110 famous sightseeing places in Kyoto city (Fig. 4).

(4) After the button "Send" was pressed, the system accepts the inputs from user and shows the auto-generated Haiku phrases on a typical Japanese-style color pattern which represents the same season described in Haiku Kigo. (Fig.5).

(5) If the user does not like the auto-generated Haiku, the user can choose regeneration with same inputs by pressing "Retry" button or do the modification by himself / herself.

(6) If the user satisfies with the generated Haiku, the user can press "Ok" button, so that the system will save the result into user-note database and show the representative photo of the sightseeing place chosen by the user with address information (Fig. 6).

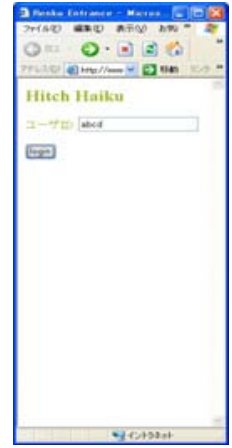

Fig. 2. Interface of user login

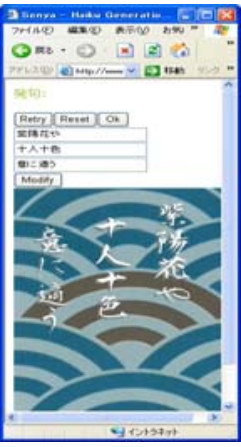

Fig. 5. Generated haiku phrases on a Japanese-style picture

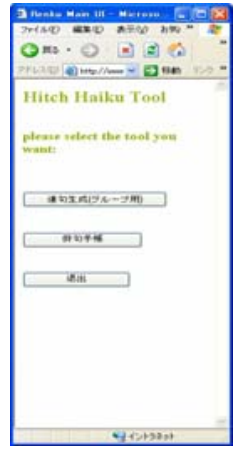

Fig. 3. Selection of functionality provided by Hitch Haiku System

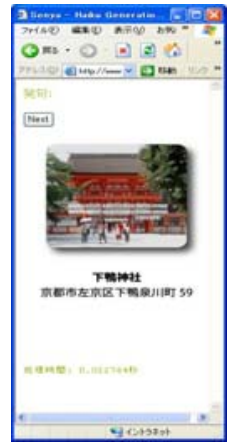

Fig. 6. Representative photo of user chosen place

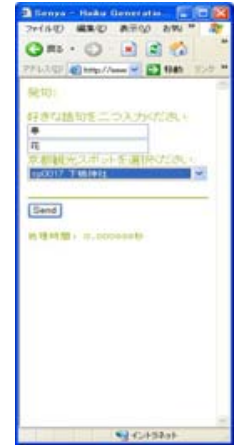

Fig. 4. Input the favorite two words and choose the spot place of sightseeing place in Kyoto

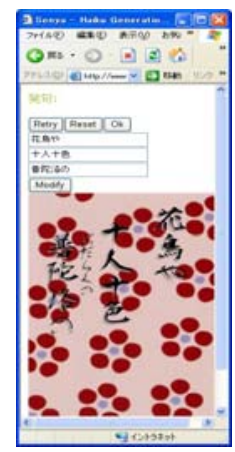

Fig. 7. An example show the ruby for some phrase difficult to read 


\section{Conclusion}

A new interactive Renku generation support system has been developed to help user enjoy the sightseeing in Kyoto city by using mobile phone, which is based on our previous interactive Haiku composition support system - Hitch Haiku. The theme of both researches is cultural computing. In order to get the better quality of Renku verses, we abandon the Kireji attachment step in the old Hitch Haiku system and mainly apply the association method to hitch the meaning of adjunct phrase tightly. In the other hand, to improve the cultural characteristics representation, the new Hitch Haiku system apply the Haiku thesaurus word to generate the Renku phrase so that the generated phrase can embed the feature of user chosen sightseeing place. Moreover, the new system adopts the Japanese-style color pattern as the background to show the auto-generated Renku verse to enhance the visual effect. In particular, the season that the selected color pattern represented is consentient to the Kigo of the generated Renku verse.

\section{References}

1. Tosa, N., Matsuoka, S.: ZENetic Computer: Exploring Japanese Culture. Leonardo, vol. 39(3), pp. 205-211. MIT Press, Cambridge(2006)

2. Matsuoka, S.: 1000 Books and 1000 Nights. Editorial Engineering Laboratory, http://www.isis.ne.jp/mnn/senya/senya.html

3. Wikipedia: Haiku, http://en.wikipedia.org/wiki/Haiku

4. Matsuo, B.: A Haiku Journey -Basho's Narrow Road to a Far Province-. Kodansha International Ltd. translated by Dorothy Britton (1974)

5. Lutz, T.: Stochastische text. Augenblick 4(1), 3-9 (1959)

6. Masterman, M.: Computerrized Haiku. Cybernetics, 175-183 (1971)

7. http://digitalmedia.risd.edu/billseaman/workMajor.php

8. Wilson, S.: Information Arts, pp. 668-671. MIT Press, Cambridge (2002)

9. Weizenbaum, J.: Eliza - a computer program for the study of natural language communication between man and machine. Communications of the ACM 9(1), 36-45 (1966)

10. Matsuoka, S.: Intelligent Editorial Engineering. Asahi Shinbun Publisher (2001)

11. Tosa, N., Obara, H., Minoh, M.: Hitch haiku: An interactive supporting system for composing haiku poem. In: Stevens, S.M., Saldamarco, S.J. (eds.) ICEC 2008. LNCS, vol. 5309, pp. 209-216. Springer, Heidelberg (2008) 